\title{
Scaling Limit for a Mechanical System of Interacting Particles
}

\author{
Kôhei Uchiyama \\ Department of Applied Physics, Tokyo Institute of Technology, Meguro-ku, Tokyo 152, Japan
}

Received: 23 November 1994/Accepted: 1 August 1995

\begin{abstract}
A system of a large number of classical particles moving on a onedimensional segment with virtually reflecting boundaries is studied. The particles interact with one another through repulsive pair-potential forces and are subjected to resistance proportional to their velocities. Because of the latter it is only the number of particles that is conserved under the evolution of the system. It is proved that in the hydrodynamic limit of diffusion type scaling the normalized counting measure of particle locations converges and its limiting density is governed by a non-linear diffusion equation which in typical cases is of porous media equation type.
\end{abstract}

\section{Introduction}

This paper concerns the problem of a hydrodynamic limit for a system of a large number of particles that move on a one-dimensional segment according to a classical equation of motion. Particles interact by exerting upon each other repulsive potential forces given by a common pair-potential function that is unbounded at zero and may have an infinite range of influence. Also at both ends of the segment there act potential forces that repel particles approaching one of the endpoints and keep them in between. In addition the medium exerts on each particle a damping force, "resistance," whose magnitude is proportional to the velocity of the particle, so that nothing except the number of particles is conserved under the evolution of the system. We take the hydrodynamic scaling limit for the normalized counting measure of particle locations in the system and prove that its limiting density is governed by a non-linear diffusion equation that in typical cases is of porous media equation type.

An approach that has been recently developed for studying the hydrodynamic limits of stochastic systems is applied to the present model. Although our system is deterministic, the application goes well owing to the dissipative effect of resistance and the fact that our system is essentially of gradient type. The idea for the general 
framework of the proof comes from Guo-Papanicolaou-Varadhan [GPV] and those for technical devices from Varadhan [V], both of them dealing with stochastic systems. [V] as well as [GPV] employs a method that is based on an entropy bound extensively, a method not available for deterministic models. However, on the one hand, once it is established that the local states of the system converges in spacetime average to an equilibrium state, the entropy bound can be dispensed with for the rest, especially for strong convergence of local density fields (the two-blocks estimate), if the system is of gradient type and the spin values are non-negative, as noted by Suzuki-Uchiyama [SU] and Uchiyama [U]. On the other hand, the equilibrium states assume a rather simple structure because of the existence of resistance: in fact, every translation invariant equilibrium state for the infinite system to the present model is degenerate at a steady state that is made up of phases such that particles stand still with equal successive spacings. This result is established by Lang $[\mathrm{L}]$ under a certain moment condition on the spacing. That condition however is difficult to check in our case, and we shall show it is dispensable.

We shall verify the local equilibrium (in the sense mentioned above) by computing the limit, under the hydrodynamic time scaling, of the time derivative of a certain functional of phases of the system. The rest of the proof is similar to that of $[\mathrm{SU}]$ and $[\mathrm{U}]$ except for some technical details: at several points nontrivial modifications must be done, necessitated for dealing with the unbounded potential function having a long tail and, in addition, with the boundary term.

A stochastic version of the present system has been investigated by OllaVaradhan $[\mathrm{OV}]$ in which independent white noises are added as random forces acting on particles. Their pair potential is smooth and has compact support. Some of our key estimates (especially the two-blocks estimate) technically may be viewed as an extension of theirs to unbounded potentials.

For systems of Newtonian particles (without damping term) the hydrodynamic limit is obtained in [BDS] in the case of hard rods (moving on the line) and discussed in [S] in the case when only nearest neighbour particles (moving on a circle) interact by a hard core potential of finite range.

Mürmann $[\mathrm{M}]$ also investigates the hydrodynamic limit of a model closely related to the present one: it deals with a nearest neighbour interaction model of deterministic dynamics in which the velocity, instead of the acceleration, of a particle is given as the gradient of a potential, with the potential function similar to ours. For such a model there are two Lyapunov functionals, i.e., the kinetic and the potential energies taken separately, which are the main tools for the study of the hydrodynamic limit in $[\mathrm{M}]$, whereas for ours there is only one Lyapunov functional, the total energy. An infinite particle system of similar nearest-neighbour interaction is studied in [F, PS].

\section{The Model and Main Result}

Consider a system of $N$ Newtonian particles of unit mass moving on the one dimensional open segment $(0, N)$ such that each particle is subjected to the resistance equal in value to its momentum, interacts with the other particles through a pair potential and is repelled with potential forces exerted by the two "walls" at 0 and 
$N$. The equation of motion for the system is written as follows:

$$
\begin{gathered}
\frac{d}{d t} q_{i}(t)=p_{i}(t), \\
\frac{d}{d t} p_{i}(t)=-p_{i}(t)-\sum_{j \neq i} U^{\prime}\left(q_{i}(t)-q_{j}(t)\right) \\
-W_{-}^{\prime}\left(q_{i}(t)\right)-W_{+}^{\prime}\left(q_{i}(t)-N\right),
\end{gathered}
$$

where $q_{i}(t)$ and $p_{i}(t)$ denote, respectively, the location and the momentum for the $i^{\text {th }}$ particle at time $t \geqq 0$ and $U^{\prime}, W_{ \pm}^{\prime}$ the derivatives of functions $U, W_{ \pm}$. The pair potential function $U(x)$, defined for $x \in \mathbf{R}-\{0\}$, is supposed to be continuously differentiable and satisfy

$$
\begin{aligned}
& U(x)=U(-x) ; \quad U(x) \geqq 0 ; \quad U(0+)=\infty ; \quad U(\infty)=0 ; \\
& U(x) \text { is strictly convex on } 0<x<R, \\
& \text { where } R:=\sup \{x>0 \mid U(x)>0\} \leqq \infty ; \\
& \qquad \int_{1}^{\infty} U(x) d x<\infty ; \\
& \qquad \liminf _{\delta \downarrow 0} \frac{1}{\psi(\delta)} \inf _{0<x<\delta} \psi(x)>0
\end{aligned}
$$

where

$$
\psi(x):=-x U^{\prime}(x), \quad x \neq 0 .
$$

From (1.2) and (1.3) it follows that $\psi(x) \geqq 0$, i.e., the pair interaction is repulsive. The assumptions (1.2) through (1.4) are fairly natural for our main result to hold $((1.3)$ is needed for showing ergodicity of the equilibrium states: see Theorem 3 below), while the assumption (1.5) may be technical although hardly restrictive; it implies in particular that $\psi(x)$ either diverges to infinity or is bounded off both zero and infinity as $x \rightarrow 0$.

For the wall potentials $W(x)=W_{-}(x)$ or $W_{+}(-x), x>0$, we suppose

$$
\begin{gathered}
W(0+)=\infty, \\
W^{\prime}(x) \leqq 0, \quad \lim _{x \rightarrow \infty} x W^{\prime}(x)=0 .
\end{gathered}
$$

The initial configuration $q_{i}=q_{i}(0), i=1,2, \ldots, N$, is always supposed to be such that $q_{i} \neq q_{j}$ if $i \neq j$ and $0<q_{i}<N$ for every $i$. The assumption $U(0+)=\infty$ together with (1.6a) guarantees that this condition for the initial configuration is preserved for all later times. The conditions (1.2) through (1.6) are assumed throughout the paper except in Theorem 4 of Sect. 4.

We are interested in a macroscopic picture for the particle configuration $\left(q_{i}(t)\right)_{i=1}^{N}$, which is to be viewed under diffusion scaling. Let us introduce the macroscopic position variables

$$
x_{i}(t)=\frac{1}{N} q_{i}\left(N^{2} t\right)
$$


and the normalized counting measure

$$
\alpha_{t}^{N}(d \theta)=\frac{1}{N} \sum_{i=1}^{N} \delta_{x_{i}(t)}(d \theta), \quad \theta \in(0,1):
$$

for an open subset $A \subset(0,1)$ it takes the value $\alpha_{t}^{N}(A)=N^{-1} \#\left\{i \mid x_{i}(t) \in A\right\}$. Our main theorem that follows asserts that the limiting density for $\alpha_{t}^{N}$ as $N \rightarrow \infty$ is characterized by the following evolution equation:

$$
\begin{gathered}
\frac{\partial}{\partial t} \rho(\theta, t)=\frac{\partial^{2}}{\partial \theta^{2}} P(\rho(\theta, t)), \quad 0<\theta<1, t>0, \\
\frac{\partial}{\partial \theta} \rho(0, t)=\frac{\partial}{\partial \theta} \rho(1, t)=0, \quad t>0,
\end{gathered}
$$

where $P$ is a function of one variable $u \geqq 0$ defined by

$$
P(u):=-\sum_{k=1}^{\infty} k U^{\prime}(k / u), \quad u>0
$$

and $P(0)=0$. The infinite sum here converges due to the assumption (1.4), which is equivalent to $\int_{1}^{\infty} \psi(x) d x<\infty$. The function $P$ may be naturally interpreted as pressure (see Remark 1 in Sect. 6); clearly $P$ is continuous and non-decreasing.

To state the theorem we need the following bound for the total energy of the initial phase $\left(p_{i}, q_{i}\right)_{i=1}^{N}:$ as $N \rightarrow \infty$,

$$
\frac{1}{2} \sum_{i=1}^{N} p_{i}^{2}+\frac{1}{2} \sum_{i, j(\neq)} U\left(q_{i}-q_{j}\right)+\sum_{i=1}^{N}\left[W_{-}\left(q_{i}\right)+W_{+}\left(q_{i}-N\right)\right]=o\left(N^{3}\right),
$$

where the second sum extends to all ordered pairs $(i, j)$ with $i \neq j, 1 \leqq i, j \leqq N$. This condition, allowing the initial energy per particle to become large up to $o\left(N^{2}\right)$, may appear very weak, but actually it turns out to be the right one for the present issue (cf. Remark 2 in Sect. 6). We will regard $\alpha_{t}^{N}$ as an element of the space of all probability measures on the closed interval $[0,1]$, which space we endow with the topology of weak convergence: according to it a sequence $\left(\alpha^{N}\right)_{N}$ in this space converges to an element $\alpha$ if and only if $\alpha^{N}(J) \rightarrow \alpha(J)$ as $N \rightarrow \infty$ for every continuous function $J$ on $[0,1]$, where $\alpha(J)$ stands for the integral of $J$ by $\alpha$.

Theorem 1. Suppose the sequence of initial phases $\left(p_{i}, q_{i}\right)_{i=1}^{N}=\left(p_{i}(0), q_{i}(0)\right)_{i=1}^{N}$, $N=1,2, \ldots$, satisfies (1.9). If $\alpha_{0}^{N}$ converges to a probability measure $\alpha_{0}$ on $[0,1]$, then $\alpha_{t}^{N}$ converges uniformly for $t<T$ for each $T<\infty$, the limit measure is absolutely continuous relative to the Lebesgue measure $d \theta$, and the density, $\rho(\theta, t)$ say, is a unique weak solution of the non-linear boundary value problem (1.8) with the initial condition

$$
\rho(\theta, t) d \theta \text { converges to } \alpha_{0} \text { as } t \downarrow 0 \text {. }
$$

The behaviour of $P(\rho)$ near $\rho=0$ is determined by that of $U(x)$ at $x=\infty$. In particular if $U^{\prime}(x) \sim C x^{-m}(m>2)$ as $x \rightarrow \infty$, then $P(\rho) \sim\left[\sum_{k \geqq 1} k^{1-m}\right] C \rho^{m}$ as $\rho \downarrow 0$, so that Eq. (1.8) assumes virtually the same form as the equation of porous medium flow; according to that theory (cf. [A], e.g.) and in view of Theorem 1, the 
macroscopic density for the system (1.1) therefore has a finite speed of propagation. By the way $P(\rho)$ behaves roughly like $\rho \int_{1 / \rho}^{\infty} \psi(x) d x$ as $\rho \rightarrow \infty$.

A heuristic argument for the derivation of (1.8) may proceed as follows. A direct computation together with some formal manipulation will deduce from (1.1)

$$
\alpha_{t}^{N}(J)-\alpha_{0}^{N}(J)=\int_{0}^{t} \frac{1}{2 N} \sum_{i=1}^{N} \sum_{j \neq i} \psi\left(N\left(x_{i}(s)-x_{j}(s)\right)\right) J^{\prime \prime}\left(x_{i}(s)\right) d s+o(1),
$$

where $J$ is a smooth test function that is constant near each of the boundary points 0 and 1 (because of this boundary condition the wall-potential terms disappear). For the identification of the inner sum on the right side we turn back to the microscopic variables $q_{i}\left(N^{2} s\right)=N x_{i}(s)$. Equilibrium states for the infinite system corresponding to (1.1) are steady states in which each particle stands still in complete balance of forces exerted on it by the other particles; and any steady state must be a configuration of equal spacing, of which the common span is necessarily equal to the reciprocal of its density. By the damping effect due to the resistance working in (1.1) velocities would get very small after such long time as of order $N^{2}$, so that the equilibrium state must be locally built up in the limit under our scaling. If there exists a limiting density $\rho(\theta, s)$, this would allow one to replace $\sum_{j \neq i} \psi\left(N\left(x_{i}(s)-x_{j}(s)\right)\right)$ by $2 \sum_{k \geqq 1} \psi\left(k / \rho\left(x_{i}(s), s\right)\right)$, which, by passing to the limit in (1.11) and noticing $P(\rho)=\rho \sum_{k \geqq 1} \psi(k / \rho)$, immediately yields the weak form of (1.8).

For this story to be justified, the following theorem (an extension of corresponding results in [OV, $\mathrm{V}$ and $\mathrm{U}]$ essentially to an unbounded potential) is fundamental at least in the present approach.

Theorem 2. Under the same assumption as in Theorem 1 there exists a continuous even function $\omega(x), x \in \mathbf{R}-\{0\}$ such that $\omega \geqq 0 ; \omega(x)$ approaches, as $x \rightarrow 0, a$ positive number or infinity according to whether $\psi$ is bounded or unbounded (near zero); and

$$
\int_{0}^{T} \sum_{i=1}^{N} \sum_{j \neq i} \sum_{k \neq i} \psi\left(N\left(x_{i}(t)-x_{j}(t)\right)\right) \omega\left(N\left(x_{i}(t)-x_{k}(t)\right)\right) d t<C N,
$$

where the constant $C$ depends on $T$ but not on $N$.

Theorem 2 will show that $(1.11)$ is valid and $\alpha_{t}^{N}, N=1,2, \ldots$, regarded as continuous measure-valued functions of $t \leqq T$, constitute a relatively compact set; moreover any limiting measure is absolutely continuous relative to the Lebesgue measure (see Corollary 1 of Sect. 3). Another, more essential application of Theorem 2 will be made, via Corollary 2 of Sect. 3, for deriving strong convergence of $\alpha_{t}^{N}$ (the two-blocks estimate) in Sect. 5.

What we need as the local equilibrium statement as mentioned in the second half of the heuristic argument given above is formulated in the next theorem. Let $\mu^{N}(d \mathbf{q})$ be the probability measure on the space of configurations $\mathbf{q}=\left(q_{i}\right)_{i=1}^{N}$ on $\mathbf{R}$ defined by

$$
\mu^{N}(H)=\frac{1}{T} \int_{0}^{T} d s \int_{0}^{1} H\left(N \mathbf{x}^{\theta}(s)\right) d \theta,
$$

where $\mathbf{x}^{\theta}$ stands for the configuration $\mathbf{x}=\left(x_{i}\right)_{i=1}^{N}$ viewed from $\theta: \mathbf{x}^{\theta}=\left(x_{i}-\theta\right)_{i=1}^{N}$ and $H=H(\mathbf{q})$ ranges over bounded, continuous local functions of locally finite configurations on $\mathbf{R}$. 
Theorem 3. Suppose that the same assumption as in Theorem 1 is fulfilled. Then any limit point $\mu$ of $\mu^{N}$ as $N \rightarrow \infty$ must be of the form

$$
\mu=\mu^{o}+\int_{0}^{R} \Phi_{\rho} \lambda(d \rho), \quad \Phi_{\rho}(d \mathbf{q}):=\rho \int_{0}^{1 / \rho} \delta_{\mathbf{e}(1 / \rho, \theta)}(d \mathbf{q}) d \theta .
$$

Here $\mu^{o}=0$ if $R=\infty$ and $\mu^{o}$ is a measure relative to which no particle is located within a range of potentials of the other particles a.e. if $R<\infty ; \lambda$ is a measure on $[0, R)$ such that $\int \rho \lambda(d \rho) \leqq 1 ; \mathbf{e}(r, \theta), r>0$, stands for $(\ldots,-r+\theta, \theta, r+\theta, 2 r+$ $\theta, \ldots)$, the configuration of particles on $\mathbf{R}$ with equal successive spacings of common span $r$ and having a particle at $\theta$; and $\Phi_{0}$ is understood to concentrate on the empty configuration.

The proof of Theorem 3 is based on a strengthening of a result by Lang [L].

Theorems 2 and 3 are proved in Sect. 3 and 4, respectively. The proof of Theorem 1 is given in Sect. 5. In Sect. 6 we present three remarks: the first one explains why $P(\rho)$ is pressure; the other two answer the illuminative question of what happens when the integrability condition (1.4) or the initial energy bound (1.9) is violated.

\section{A Bound of the Kinetic Energy}

We isolate this short section in which we note that for the system (1.1) the kinetic energy per particle approaches zero in the time average as $N \rightarrow \infty$ on account of assumption (1.9), the result being basic in all the later sections. Put $v_{i}(t)=p_{i}\left(N^{2} t\right)$. Then the equation of motion (1.1) becomes

$$
\begin{gathered}
\frac{d}{d t} x_{i}(t)=N v_{i}(t) \\
\frac{d}{d t} v_{i}(t)=-N^{2} v_{i}(t)-N^{2} \sum_{j \neq i} U^{\prime}\left(N\left(x_{i}(t)-x_{j}(t)\right)\right) \\
\left.-N^{2} W_{-}^{\prime}\left(N x_{i}(t)\right)-N^{2} W_{+}^{\prime}\left(N\left(x_{i}(t)-1\right)\right)\right) .
\end{gathered}
$$

For the total energy

$$
\begin{aligned}
\mathscr{E}^{N}(t):= & \frac{1}{2} \sum_{i=1}^{N} v_{i}^{2}(t)+\frac{1}{2} \sum_{i, j(\neq)} U\left(N\left(x_{i}(t)-x_{j}(t)\right)\right) \\
& \left.+\sum_{i=1}^{N}\left[W_{-}\left(N x_{i}(t)\right)+W_{+}\left(N\left(x_{i}(t)-1\right)\right)\right)\right],
\end{aligned}
$$

we calculate its derivative to obtain

$$
\frac{d}{d t} \mathscr{E}^{N}(t)=-N^{2} \sum_{i=1}^{N} v_{i}^{2}(t) \leqq 0
$$

in particular $\mathscr{E}^{N}(t) \leqq \mathscr{E}^{N}(0)$ and

$$
\int_{0}^{T} \frac{1}{N} \sum_{i=1}^{N} v_{i}^{2}(t) d t \leqq \frac{1}{N^{3}} \mathscr{E}^{N}(0)
$$

of which the right side goes to zero as $N \rightarrow \infty$ under (1.9). 


\section{Estimation of $\sum \psi\left(N\left(x_{i}-x_{j}\right)\right)$}

This section is divided into four parts. The first three parts are devoted to the proof of Theorem 2, while in the last one we state three corollaries of Theorem 2 and its proof. The idea of the proof is essentially the same as that for the corresponding one in [V]. Our potential function, however, is not bounded, nor necessarily of compact support as in [V], and this necessitates a significant modification of the proof.

3.1. Let $g=g(x), x \neq 0$, be an even, continuous function such that $g$ is nonincreasing on $(0, \infty)$,

$$
\begin{aligned}
g(x) & =\frac{a}{\sqrt{|x|}}, & & 0<|x|<\frac{1}{2} \\
& =0, & & |x|>1,
\end{aligned}
$$

where $a$ is a positive constant (the precise form of $g$ as given above is unimportant) and

$$
\int_{-\infty}^{\infty} g(x) d x=1
$$

Let $h(x), x \in \mathbf{R}$ be a smooth non-negative even function such that $\int h(x) d x=1$ and $h(x)=0$ for $|x|>1$. We write $g_{N}(x)=N g(N x)$ and define a function $G_{(N)}$ on $\mathbf{R}$ by

$$
G_{(N)}(x)=\int_{-\infty}^{x} d y \int_{-\infty}^{y}\left(g_{N}(u)-h(u)\right) d u
$$

so that

$$
G_{(N)}^{\prime \prime}(x)=g_{N}(x)-h(x) .
$$

Both $\left|G_{(N)}\right|$ and $\left|G_{(N)}^{\prime}\right|$ are bounded by 1 , and vanish for $|x|>1$. Put

$$
B_{N}(x):=N W_{-}^{\prime}(N x)+N W_{+}^{\prime}(N(x-1)), \quad|x|<1 .
$$

Then for $G=G_{(N)}$, Eq. (2.1) yields

$$
\begin{aligned}
\frac{d}{d t} \sum_{i, j(\neq)} G^{\prime}\left(x_{i}-x_{j}\right) v_{i}= & N \sum_{i, j(\neq)} G^{\prime \prime}\left(x_{i}-x_{j}\right) v_{i}\left(v_{i}-v_{j}\right)-N^{2} \sum_{i, j(\neq)} G^{\prime}\left(x_{i}-x_{j}\right) v_{i} \\
& -N \sum_{i} \sum_{k \neq i} G^{\prime}\left(x_{i}-x_{k}\right)\left[\sum_{j \neq i} N U^{\prime}\left(N\left(x_{i}-x_{j}\right)\right)\right] \\
& -N \sum_{i} \sum_{j \neq i} G^{\prime}\left(x_{i}-x_{j}\right) B_{N}\left(x_{i}\right) .
\end{aligned}
$$

Here (and below) the time variable is suppressed from functions $x_{i}(t), v_{i}(t)$, etc. for the sake of brevity. In the third term on the right side above we may replace $G^{\prime}\left(x_{i}-x_{k}\right)$ by $\frac{1}{2}\left[G^{\prime}\left(x_{i}-x_{k}\right)-G^{\prime}\left(x_{j}-x_{k}\right)\right]$ if $j \neq k$, since $U^{\prime}$ is odd. Noticing $G_{(N)}^{\prime}$ is also odd, we see that the first term on the right side equals $\frac{1}{2} N \sum\left(v_{i}-v_{j}\right)^{2} G^{\prime \prime}\left(x_{i}-x_{j}\right)$ and the second $-\frac{1}{2} N(d / d t) \sum G\left(x_{i}-x_{j}\right)$. Integrating both sides of the resulting 
equality on $[0, T]$ and then dividing them by $N^{3}$, we obtain after rearrangement of terms

$$
\begin{aligned}
& \frac{1}{2 N^{2}} \int_{0}^{T} \sum_{i, j, k(\neq)}\left[-N U^{\prime}\left(N\left(x_{i}-x_{j}\right)\right)\right]\left[G^{\prime}\left(x_{i}-x_{k}\right)-G^{\prime}\left(x_{j}-x_{k}\right)\right] d t \\
& +\frac{1}{N^{2}} \int_{0}^{T} \sum_{i, j(\neq)}\left[-N U^{\prime}\left(N\left(x_{i}-x_{j}\right)\right)\right] G^{\prime}\left(x_{i}-x_{j}\right) d t \\
& =\left.\frac{1}{N^{3}} \sum_{i, j(\neq)} v_{i} G^{\prime}\left(x_{i}-x_{j}\right)\right|_{t=0} ^{T}+\left.\frac{1}{2 N^{2}} \sum_{i, j(\neq)} G\left(x_{i}-x_{j}\right)\right|_{t=0} ^{T} \\
& -\frac{1}{2 N^{2}} \int_{0}^{T} \sum_{i, j(\neq)}\left(v_{i}-v_{j}\right)^{2} G^{\prime \prime}\left(x_{i}-x_{j}\right) d t \\
& +\frac{1}{N^{2}} \int_{0}^{T} \sum_{i, j(\neq)} B_{N}\left(x_{i}\right) G^{\prime}\left(x_{i}-x_{j}\right) d t,
\end{aligned}
$$

where the triple sum on the left side extends over all ordered triplets with three components different from one another. In view of (2.2) and the inequality

$$
\frac{1}{N^{2}} \sup _{t} \sum\left|v_{i}(t)\right| \leqq \sup _{t}\left[N^{-3} \sum v_{i}^{2}(t)\right]^{1 / 2} \leqq\left[N^{-3} \mathscr{E}^{N}(0)\right]^{1 / 2},
$$

the sum of the first two terms on the right side of (3.1) may be estimated from above by $2 \sqrt{\mathscr{E}^{N}(0) / N^{3}}+\|G\|_{\infty}$. Since $-G_{(N)}^{\prime \prime}(x) \leqq h(x)$, the third term is bounded above by $2\|h\|_{\infty} \mathscr{E}^{N}(0) / N^{3}$. For estimating the boundary term we make use of the relation (5.3) given in Sect. 5 ( clearly its verification is made independently of any result obtained in this section). Taking $J(x)=\left(x-\frac{1}{2}\right)^{2}$ in (5.3) and applying (2.2) and the assumption (1.6b) we see

$$
\int_{0}^{T} \sum_{i=1}^{N}\left|B_{N}\left(x_{i}\right)\right| d t \leqq N+C_{1} \frac{\mathscr{E}^{N}(0)}{N^{2}}+2 \int_{0}^{T} \sum_{i, j(\neq)} \psi\left(N\left(x_{i}-x_{j}\right)\right) d t
$$

Thus from condition (1.9) or, what is the right one here, $\mathscr{E}^{N}(0)=O\left(N^{3}\right)$ it follows that

$$
\begin{aligned}
& \frac{1}{2 N^{2}} \int_{0}^{T} \sum_{i, j, k(\neq)}\left[-N U^{\prime}\left(N\left(x_{i}-x_{j}\right)\right)\right]\left[G^{\prime}\left(x_{i}-x_{k}\right)-G^{\prime}\left(x_{j}-x_{k}\right)\right] d t \\
& +\frac{1}{N^{2}} \int_{0}^{T} \sum_{i, j(\neq)}\left[-N U^{\prime}\left(N\left(x_{i}-x_{j}\right)\right)\right] G^{\prime}\left(x_{i}-x_{j}\right) d t \\
& \leqq C_{2}+\frac{2}{N} \int_{0}^{T} \sum_{i, j(\neq)} \psi\left(N\left(x_{i}-x_{j}\right)\right) d t .
\end{aligned}
$$

The remainder of the proof of Theorem 2 concerns inequalities that are valid for an arbitrary configuration $\left(x_{1}, x_{2}, \ldots, x_{N}\right)$ and have nothing to do with the dynamics (1.1). 
3.2. Let us estimate the left side of (3.4) from below. For simplifying the arguments that follow we assume $R>1$. To begin with we notice the following two trivial inequalities:

$$
\begin{gathered}
\frac{G^{\prime}\left(x_{i}-x_{k}\right)-G^{\prime}\left(x_{j}-x_{k}\right)}{x_{i}-x_{j}}=\frac{1}{x_{i}-x_{j}} \int_{x_{j}}^{x_{1}}\left[g_{N}-h\right]\left(y-x_{k}\right) d y \\
\geqq \frac{1}{x_{i}-x_{j}} \int_{x_{j}}^{x_{1}} g_{N}\left(y-x_{k}\right) d y-\|h\|_{\infty} ; \\
\frac{G^{\prime}\left(x_{i}-x_{j}\right)}{x_{i}-x_{j}} \geqq g_{N}\left(x_{i}-x_{j}\right)-\|h\|_{\infty} .
\end{gathered}
$$

It is here in the proof of the next lemma that we need assumption (1.5). We put

$$
\psi^{*}(x)=\psi(x) \chi\{|x| \leqq 1 / 4\}
$$

where $\chi\{|x| \leqq a\}=1$ or 0 according as $|x| \leqq a$ or $|x|>a$.

Lemma 1. There exists an even function $\omega(x), x \in \mathbf{R}-\{0\}$ such that $\omega \geqq 0 ; \omega(x)$ is positive and continuous for $0<|x|<1 / 4 ; \omega(x)$ converges to a positive number or diverges to infinity as $x \rightarrow 0$ according as $\psi^{*}$ is bounded or unbounded; and

$$
\sum_{i, j, k(\neq)} \psi\left(N\left(x_{i}-x_{j}\right)\right) \frac{1}{x_{i}-x_{j}} \int_{x_{j}}^{x_{i}} g_{N}\left(y-x_{k}\right) d y \geqq N \sum_{i, j, k(\neq)} \psi^{*}\left(N\left(x_{i}-x_{j}\right)\right) \omega\left(N\left(x_{i}-x_{k}\right)\right)
$$

Proof. Since $(y-x)^{-1} \int_{x}^{y} g_{N}(u-z) d u \geqq \min \left\{g_{N}(x-z), g_{N}(y-z)\right\}$, the inequality required in the lemma is implied by

$$
\begin{gathered}
\max _{\pi \in \subseteq(3)}\left[\psi\left(q_{\pi(i)}-q_{\pi(j)}\right) \min \left\{g\left(q_{\pi(i)}-q_{\pi(k)}\right), g\left(q_{\pi(j)}-q_{\pi(k)}\right)\right\}\right] \\
\geqq 6 \max _{\pi \in \subseteq(3)}\left[\psi^{*}\left(q_{\pi(i)}-q_{\pi(j)}\right) \omega\left(q_{\pi(i)}-q_{\pi(k)}\right)\right],
\end{gathered}
$$

where $\mathfrak{S}(3)$ is the permutation group of three letters $i, j, k$ and $q_{i}, q_{j}$ and $q_{k}$ are arbitrary three real numbers different from one another. If we define $\tilde{\omega}(x), 0<x \leqq$ $1 / 2$, by

$$
\tilde{\omega}(x)=\inf _{0<u<x} \tilde{g}(u), \quad \tilde{g}(x)=\psi(x) \inf _{u: x \leqq u \leqq 1 / 2} \frac{g(u)}{\psi(u)}
$$

(recall we have supposed $R>1$ ), then $\tilde{\omega}$ is non-increasing; $\tilde{\omega}(x)$ approaches infinity or a positive number as $x \downarrow 0$ according as $\psi^{*}$ is unbounded or bounded by virtue of assumption (1.5); moreover if $0<s \leqq t \leqq 1 / 4$,

$$
\psi(s) g(s+t) \geqq \frac{1}{\sqrt{2}} \max \{\psi(s+t) \tilde{\omega}(s), \psi(t) \tilde{\omega}(s), \psi(s) \tilde{\omega}(t)\}
$$

Finally define $\omega(x)=(6 \sqrt{2})^{-1} \tilde{\omega}(|x|) \chi\{|x|<1 / 4\}$. A little reflection then shows that (3.6) follows from (3.7), finishing the proof of Lemma 1. 

clude:

Substituting the inequality (3.5) and that given in Lemma 1 into (3.4) we con-

$$
\begin{aligned}
& \frac{1}{N} \int_{0}^{T} \sum_{i, j(\neq)}\left[\psi^{*}\left(N\left(x_{i}-x_{j}\right)\right) \sum_{k \neq i} \omega\left(N\left(x_{i}-x_{k}\right)\right)\right] d t \\
& \quad \leqq C+\left(4+\|h\|_{\infty}\right) \frac{1}{N} \int_{0}^{T} \sum_{i, j(\neq)} \psi\left(N\left(x_{i}-x_{j}\right)\right) d t .
\end{aligned}
$$

3.3. We wish to dominate the integral on the right side of (3.8) by a small fraction of that on the left side. We continue to assume $R>1$ and choose the function $\omega$ in Lemma 1 so that $\omega \leqq g$ as in the proof of it.

Lemma 2. There are constants $C_{1}$ and $C_{2}$ that depend only on $\psi$ such that

$$
\sum_{i, j(\neq)} \psi\left(N\left(x_{i}-x_{j}\right)\right) \leqq C_{1} N+C_{2} \sum_{i, j(\neq)} \psi^{*}\left(N\left(x_{i}-x_{j}\right)\right) \text {. }
$$

Proof. For $\ell>0$ put

$$
n_{\ell}(\theta)=n_{N, \ell}(\theta ; \mathbf{x}):=\#\left\{j|| x_{j}-\theta \mid \leqq \frac{\ell}{2 N}\right\} .
$$

Then, by noticing that $\sup _{a \leqq x \leqq b} \psi(x) \leqq(b / a) \psi(a)$ if $0<a<b$,

$$
\begin{aligned}
\psi_{i}^{* *} & :=\sum_{j \neq i} \psi\left(N\left(x_{i}-x_{j}\right)\right) \chi\left\{N\left|x_{i}-x_{j}\right|>1 / 4\right\} \\
& \leqq 2 \sum_{k \neq 0} \psi\left(\frac{k}{4}\right) n_{1 / 4}\left(x_{i}+\frac{k}{4 N}+\frac{k /|k|}{8 N}\right) .
\end{aligned}
$$

But for every integer $k$,

$$
\begin{aligned}
& \sum_{i} n_{1 / 4}\left(x_{i}+\frac{k}{4 N}+\frac{1}{8 N}\right) \\
& \quad \leqq \sum_{m} n_{1 / 4}\left(\frac{m}{4 N}\right)\left[n_{1 / 4}\left(\frac{m}{4 N}+\frac{k}{4 N}\right)+n_{1 / 4}\left(\frac{m}{4 N}+\frac{k-1}{4 N}\right)\right] \\
& \quad \leqq 2 \sum_{m} n_{1 / 4}^{2}\left(\frac{m}{4 N}\right) .
\end{aligned}
$$

Thus

$$
\sum_{i} \psi_{i}^{* *} \leqq 4\left[\sum_{k \neq 0} \psi\left(\frac{k}{4}\right)\right] \sum_{m} n_{1 / 4}^{2}\left(\frac{m}{4 N}\right) .
$$

On the other hand, by putting

$$
\begin{aligned}
\psi_{i}^{*}:= & \sum_{j \neq i} \psi\left(N\left(x_{i}-x_{j}\right)\right) \chi\left\{N\left|x_{i}-x_{j}\right| \leqq 1 / 4\right\}, \\
\sum_{i} \psi_{i}^{*} & \geqq\left(\inf _{|u| \leqq 1 / 4} \psi(u)\right) \sum_{i}\left(n_{1 / 2}\left(x_{i}\right)-1\right) \\
& \geqq\left(\inf _{|u| \leqq 1 / 4} \psi(u)\right)\left[\sum_{m} n_{1 / 4}^{2}\left(\frac{m}{4 N}\right)-N\right] .
\end{aligned}
$$

We can therefore find two constants $C_{1}$ and $C_{2}$ for the inequality of the lemma to hold. 
Lemma 3. For every $\delta>0$ there can be found a constant $M$ so that

$$
\sum_{i, j(\neq)} \psi\left(N\left(x_{i}-x_{j}\right)\right) \leqq N M+\delta \sum_{i, j(\neq)}\left[\psi^{*}\left(N\left(x_{i}-x_{j}\right)\right) \sum_{k \neq i} \omega\left(N\left(x_{i}-x_{k}\right)\right)\right]
$$

Proof. Put

$$
\begin{aligned}
\omega_{i}= & \sum_{j \neq i} \omega\left(N\left(x_{i}-x_{j}\right)\right), \\
\gamma(u)= & \inf \left\{\sum_{k} \omega\left(s_{k}\right) \mid\left(s_{k}\right)_{k}\right. \text { is a finite sequence } \\
& \left.\quad \text { of real numbers such that } \sum_{k} \psi^{*}\left(s_{k}\right) \geqq u\right\} .
\end{aligned}
$$

Then $\gamma(u) \uparrow \infty$ as $u \uparrow \infty$, and, since $\gamma\left(\psi_{i}^{*}\right) \leqq \omega_{i}$,

$$
\psi_{i}^{*} \chi\left\{\psi_{i}^{*} \geqq u\right\} \leqq \frac{1}{\gamma(u)} \psi_{i}^{*} \omega_{i}
$$

hence $\sum \psi_{i}^{*} \leqq N u+\sum \psi_{i}^{*} \chi\left\{\psi_{i}^{*} \geqq u\right\} \leqq N u+(1 / \gamma(u)) \sum \psi_{i}^{*} \omega_{i}$. Substituting the last inequality into Lemma 2 and choosing $u$ so large that $C_{2} / \gamma(u)<\delta$, we obtain the estimate claimed in the lemma.

Lemma 4. Let $\psi_{i}^{* *}$ be as in (3.9). Then there are constants $C_{1}$ and $C_{2}$ such that

$$
\sum_{i}\left[\psi_{i}^{* *}\right]^{2} \leqq C_{1} N+C_{2} \sum_{i, j(\neq)}\left[\psi^{*}\left(N\left(x_{i}-x_{j}\right)\right) \sum_{k \neq i} \omega\left(N\left(x_{i}-x_{k}\right)\right)\right] .
$$

Proof. Denote by $S^{N}$ the sum on the right side. As in the proof of Lemma 2 we see

$$
\begin{aligned}
S^{N} & \geqq\left(\inf _{|u| \leqq 1 / 8} \psi(u) \omega(u)\right) \sum_{i}\left(n_{1 / 4}\left(x_{i}\right)-1\right)^{2} \\
& \geqq\left(\inf _{|u| \leqq 1 / 8} \psi(u) \omega(u)\right)\left[\sum_{m} n_{1 / 8}^{3}\left(\frac{m}{8 N}\right)-6 \sum_{m} n_{1 / 4}^{2}\left(\frac{m}{4 N}\right)\right] .
\end{aligned}
$$

The second term within the square bracket on the last line being dominated by a constant multiple of $S^{N}+N$ according to (3.12) and Lemma 3, this inequality yields

$$
\sum_{m} n_{1 / 4}^{3}\left(\frac{m}{4 N}\right) \leqq C_{1}^{\prime} N+C_{2}^{\prime} S^{N}
$$

On the other hand applying the Schwarz inequality to the right side of (3.9) and then making the same argument as in (3.10) we see

$$
\begin{aligned}
\sum_{i}\left[\psi_{i}^{* *}\right]^{2} & \leqq 4 \sum_{i}\left[\sum_{k \neq 0} \psi\left(\frac{k}{4}\right)\right]\left[\sum_{k \neq 0} \psi\left(\frac{k}{4}\right) n_{1 / 4}^{2}\left(x_{i}+\frac{k}{4 N}+\frac{k /|k|}{8 N}\right)\right] \\
& \leqq 4 \cdot 4\left[\sum_{k \neq 0} \psi\left(\frac{k}{4}\right)\right]^{2} \sum_{m} n_{1 / 4}^{3}\left(\frac{m}{4 N}\right)
\end{aligned}
$$


of which, owing to (3.14), the last member is dominated by $C_{1} N+C_{2} S^{N}$ as required.

The proof of Theorem 2 is now completed in a few lines. By applying the Schwarz inequality and Lemma 4 in turn we see

$$
\begin{aligned}
& \sum_{i, j(\neq)}\left[\psi\left(N\left(x_{i}-x_{j}\right)\right) \chi\left\{N\left|x_{i}-x_{j}\right|>1 / 4\right\} \sum_{k \neq i} \omega\left(N\left(x_{i}-x_{k}\right)\right)\right] \\
& \leqq C_{3}+C_{4} \sum_{i, j(\neq)}\left[\psi^{*}\left(N\left(x_{i}-x_{j}\right)\right) \sum_{k \neq i} \omega\left(N\left(x_{i}-x_{k}\right)\right)\right] .
\end{aligned}
$$

The asserted estimate is obtained by applying this one after substituting that of Lemma 3 into (3.8).

3.4. We state three corollaries of Theorem 2 for convenience of later citations.

Corollary 1. For each $T>0$,

$$
\lim _{\delta \downarrow 0} \sup _{\substack{0 \leq s<t \leq T \\|t-s|<\delta}} \sup _{N} \int_{s}^{t} \frac{1}{N} \sum_{i, j(\neq)} \psi\left(N\left(x_{i}(u)-x_{j}(u)\right)\right) d u=0 .
$$

Proof. Immediate from Lemma 3 and Theorem 2.

Corollary 2. The functions $\Psi^{N}(t, i):=\sum_{j \neq i} \psi\left(N\left(x_{i}(t)-x_{j}(t)\right)\right), N=1,2, \ldots$, are uniformly integrable relative to the respective product measures $N^{-1} \sum_{j=1}^{N} \delta_{j}(\cdot) \times$ $d t \in \mathfrak{M}(\{1,2, \ldots, N\} \times[0, T])$.

Proof. In the decomposition $\Psi^{N}(\cdot, i)=\psi_{i}^{*}+\psi_{i}^{* *}$ (where $\psi_{i}^{* *}$ is defined in the proof of Lemma 2), the uniform integrability of the first term, $\psi_{i}^{*}$, follows from (3.13) and Theorem 2. As for the second term Lemma 4 and Theorem 2 together verify that its second moment is bounded; it is in particular uniformly integrable.

By making the same argument as leading to (3.11) we deduce from Theorem 2, Lemma 3 and (3.11) the following

Corollary 3. Let $\varphi$ be a continuous, integrable positive function on $\mathbf{R}$ and suppose $\varphi(y) / \varphi(x)$ is bounded on $\{|x|<|y|\}$. Then there exists a constant $C$ such that for all $N=1,2, \ldots$ and all $M \geqq 0$,

$$
\int_{0}^{T} \sum_{i, j(\neq)} \varphi\left(N\left(x_{i}(t)-x_{j}(t)\right)\right) \chi\left\{N\left|x_{i}(t)-x_{j}(t)\right| \geqq M\right\} d t \leqq C N \sum_{|k| \geqq M} \varphi(k) .
$$

\section{Local Equilibrium}

Recall $\mu^{N}$ denotes the probability measure on the set of $N$-particle configurations determined by (1.12). Since the expectation of the density of configurations under $\mu^{N}$ is (uniformly) bounded, the family $\left(\mu^{N}\right)_{N \geqq 1}$ is relatively compact in the space of probability measures on the set of locally finite configurations on $\mathbf{R}$ under the usual topology determined by the convergence of the integral of bounded, continuous local functions. In this section we prove Theorem 3, namely that any limit point of $\mu^{N}$, which is necessarily translation invariant, concentrates on configurations of equal spacing. We note that if $\mu$ is a limit point of $\mu^{N}$, then $\mu\left\{\mathbf{q} \mid q_{i} \neq q_{j}\right.$ if $\left.i \neq j\right\}=1$, as is verified by Theorem 2 . 
Lemma 5. For every limit point $\mu$ of $\mu^{N}$,

$$
\mu\left\{\mathbf{q}=\left(q_{i}\right) \mid \sum_{j \neq i} U^{\prime}\left(q_{i}-q_{j}\right)=0 \text { for all } i\right\}=1 .
$$

Proof. In a formal level the result of Lemma 5 is inferred from an identity (set $g \equiv 1$ below) obtained by calculating the right side of

$$
\left.N^{-3} \sum v_{i} \sum_{j \neq i} U^{\prime}\left(x_{i}-x_{j}\right)\right|_{t=0} ^{T}=N^{-3} \int_{0}^{T} \frac{d}{d t}\left[\sum v_{i} \sum_{j \neq i} U^{\prime}\left(x_{i}-x_{j}\right)\right] d t
$$

according to (2.1) and then passing to the limit as $N \rightarrow \infty$. In the identity however there arise singular terms that should be avoided for the latter procedure of taking the limit. In this proof we truncate them by means of two functions $g$ and $\varphi$ whose introduction we start with.

For each $K>3$, let $g(u)=g(u, K)$ be a smooth function on $\mathbf{R}$ such that

$$
g(u)=0 \quad \text { if }|u|>K ; \quad=1 \text { if }|u| \leqq K-2 ; \quad \text { and } \quad|g|,\left|g^{\prime}\right| \leqq 1 .
$$

Let $\varphi$ be a smooth function on $\mathbf{R}-\{0\}$ that satisfies the following conditions:

$$
\begin{gathered}
\left|U^{\prime}(x)\right| \leqq \varphi(x) \text { and }-x \varphi^{\prime}(x)>0 \text { for } x \neq 0 ; \quad \int_{|x|>1} \varphi(x) d x<\infty, \\
\left|\varphi^{\prime}(x)\right| \leqq \varphi(x) \text { for }|x|>1 ; \quad \text { and }\left|\varphi^{\prime}(y)\right| \leqq 2\left|\varphi^{\prime}(x)\right| \text { if }|y|>|x|>0 .
\end{gathered}
$$

Such a function $\varphi$ may be constructed by modifying $U$ as follows. The problem is how to define it for large $x$. Clearly we can suppose $R=\infty$. Put $f(x)=-U^{\prime}(x-1) /$ $U(x-1), x \geqq 2$. If $\sup f \leqq 1$, we can take $\varphi(x)=$ const $U(x-1)$ for $x \geqq 2$, since $U(x-1) \geqq-U^{\prime}(x), x \geqq 2$. If $\sup f>1$, putting $x_{o}=\inf \{x \geqq 2 \mid f(x)>1\}$, we define $\varphi(x)=U(x-1)$ for $2 \leqq x \leqq x_{o}$ and

$$
\varphi(x)=U\left(x_{o}-1\right) \exp \left\{-\left(x-x_{o}\right) / 2\right\} \quad \text { for } x_{o}<x \leqq x_{1},
$$

where $x_{1}$ is the least value of $x \geqq x_{o}$ at which $U\left(x_{o}-1\right) \exp \left\{-\left(x-x_{o}\right)\right\}$ coincides with $U(x-1)$. Necessarily $f\left(x_{1}\right) \leqq 1 / 2$ if $x_{1}<\infty$. Now starting from $x_{1}$ instead of 2 we continue the same procedure as above to define $\varphi$ on $[2, K]$ for every $K$ and hence on $[2, \infty)$. The function $\varphi$ thus defined fulfills all the requirements as is easily checked.

Let $\tilde{U}_{(\varepsilon)}^{\prime}(x)$ be a smooth approximation of $U^{\prime}(x)$ such that $\left|\tilde{U}_{(\varepsilon)}^{\prime}(x)\right| \leqq 2 \varphi(x)$, $\left|\tilde{U}_{(\varepsilon)}^{\prime \prime}(x)\right| \leqq C_{\varepsilon}\left|\varphi^{\prime}(x)\right|$ and $\tilde{U}_{(\varepsilon)}^{\prime}(x) \rightarrow U^{\prime}(x)(\varepsilon \downarrow 0)$ for each $x \neq 0$. Now, putting

$$
\begin{aligned}
& \varphi_{i}(t)=\sum_{j \neq i} \varphi\left(N\left(x_{i}(t)-x_{j}(t)\right)\right), \\
& F_{i}(t)=-\sum_{j \neq i} U^{\prime}\left(N\left(x_{i}(t)-x_{j}(t)\right)\right), \\
& \tilde{F}_{i, \varepsilon}(t)=-\sum_{j \neq i} \tilde{U}_{(\varepsilon)}^{\prime}\left(N\left(x_{i}(t)-x_{j}(t)\right)\right),
\end{aligned}
$$


we see

$$
\begin{aligned}
& \frac{1}{N^{3}} \frac{d}{d t}\left[\sum_{i=1}^{N} v_{i}(t) \tilde{F}_{i, \varepsilon}(t) g\left(\varphi_{i}(t)\right)\right]=-\frac{1}{N} \sum_{i} v_{i} \tilde{F}_{i} g\left(\varphi_{i}\right)+\frac{1}{N} \sum_{i} F_{i} \tilde{F}_{i} g\left(\varphi_{i}\right) \\
& -\frac{1}{N^{2}} \sum_{i} B_{N}\left(x_{i}\right) \tilde{F}_{i} g\left(\varphi_{i}\right)-\frac{1}{N} \sum_{i, j(\neq)} v_{i}\left(v_{i}-v_{j}\right) \tilde{U}^{\prime \prime}\left(N\left(x_{i}-x_{j}\right)\right) g\left(\varphi_{i}\right) \\
& +\frac{1}{N} \sum_{i} v_{i} \tilde{F}_{i} g^{\prime}\left(\varphi_{i}\right) \sum_{j \neq i} \varphi^{\prime}\left(N\left(x_{i}-x_{j}\right)\right)\left(v_{i}-v_{j}\right) .
\end{aligned}
$$

Here the argument $t$ and the parameter $\varepsilon$ are suppressed on the right side. We integrate both sides of this identity with respect to $t \in[0, T]$ and take the limit as $N \rightarrow \infty$. We are to show that the contribution of every term except the second one on the right side vanishes in the limit to have

$$
\lim _{N \rightarrow \infty} \int_{0}^{T} \frac{1}{N} \sum_{i} F_{i}(t) \tilde{F}_{i, \varepsilon}(t) g\left(\varphi_{i}(t) ; K\right) d t=0
$$

for every $K>0$ (with $\varepsilon$ fixed). By noticing that $\left|\tilde{F}_{i} g\left(\varphi_{i}\right)\right| \leqq 2 K$ and by making use of (2.2), (3.2), (3.3), Lemma 3 and Theorem 2, the left side as well as the first and third terms on the right side are readily disposed of to this end. For dealing with the rest, i.e., the last two terms on the right side we choose, for each $K, \delta=\delta(K)>0$ so that $\varphi(2 \delta)>K$, which is possible since $\varphi(x) \rightarrow \infty$ as $x \rightarrow 0$. Then

$$
g\left(\varphi_{i}\right)=g^{\prime}\left(\varphi_{i}\right)=0 \quad \text { if } \min \left\{x_{i}-x_{i-1}, x_{i+1}-x_{i}\right\} \leqq \frac{2 \delta}{N},
$$

where $x_{i}$ are numbered in the increasing order: $x_{1}<x_{2}<\cdots<x_{N}$ and $x_{0}=-\infty$ and $x_{N+1}=\infty$. It would be clear from the properties imposed on $\varphi$ that our task for the proof of (4.2) is reduced to showing

$$
\begin{aligned}
& \int_{0}^{T} \sum_{i} v_{i} g\left(\varphi_{i}\right) \sum_{j \neq i} \tilde{U}^{\prime \prime}\left(N\left(x_{i}-x_{j}\right)\right) v_{j}=o(N), \\
& \int_{0}^{T} \sum_{i} v_{i} \tilde{F}_{i} g^{\prime}\left(\varphi_{i}\right) \sum_{j \neq i} \varphi^{\prime}\left(N\left(x_{i}-x_{j}\right)\right) v_{j}=o(N) .
\end{aligned}
$$

(These estimates are ready to verify if we assume $\mathscr{E}^{N}(0)=o\left(N^{2}\right)$; see also (6.1).) We prove $(4.4 \mathrm{~b})$ first. Put

$$
\chi_{i, m}=\chi\left\{(m-1) \delta \leqq N x_{i}<m \delta\right\} .
$$

Then

$\left|\sum_{i} v_{i} \tilde{F}_{i} g^{\prime}\left(\varphi_{i}\right) \sum_{j \neq i} \varphi^{\prime}\left(N\left(x_{i}-x_{j}\right)\right) v_{j}\right| \leqq 2 K \sum_{i} \sum_{m} \chi_{i, m}\left|v_{i} g^{\prime}\left(\varphi_{i}\right)\right| \sum_{j \neq i}\left|\varphi^{\prime}\left(N\left(x_{i}-x_{j}\right)\right) v_{j}\right|$.

If $\chi_{i, m}=1$, i.e., $(m-1) \delta \leqq N x_{i}<m \delta$, then by the last condition imposed on $\varphi$,

$$
\left.\sum_{j \neq i}\left|\varphi^{\prime}\left(N\left(x_{i}-x_{j}\right)\right) v_{j}\right| \leqq 2 \sum_{j>i}\left|\varphi^{\prime}\left(m \delta-N x_{j}\right) v_{j}\right|+2 \sum_{j<i} \mid \varphi^{\prime}\left((m-1) \delta-N x_{j}\right)\right) v_{j} \mid .
$$


Thus the contribution of the sum over $j>i$ to the right side of (4.5) is at most

$$
\begin{aligned}
& 4 K \sum_{i} \sum_{m} \chi_{i, m}\left|v_{i} g^{\prime}\left(\varphi_{i}\right)\right| \sum_{j>i}\left|\varphi^{\prime}\left(m \delta-N x_{j}\right) v_{j}\right| \\
& \quad \leqq 8 K \sum_{k \geqq 1}\left|\varphi^{\prime}(k \delta)\right| \sum_{i} \sum_{m} \chi_{i, m}\left|v_{i} g^{\prime}\left(\varphi_{i}\right)\right| \sum_{j} \chi_{j, m+k+1}\left|v_{j}\right| .
\end{aligned}
$$

Here we have applied (4.3) again. Observe that

$$
\chi_{i, m} g^{\prime}\left(\varphi_{i}\right)=0 \text { if } \sum_{j} \chi_{j, m+k+1}>\frac{K}{\varphi((k+2) \delta)} \text { for some } k \geqq 1 .
$$

Then by applying the Schwarz inequality both to the double sum over $i, m$ and to the sum over $j$ we can further dominate the sum on the right side of (4.6) by

$$
\begin{aligned}
& \sum_{k \geqq 1} \frac{\left|\varphi^{\prime}(k \delta)\right| \sqrt{K}}{\sqrt{\varphi((k+2) \delta)}} \sqrt{\sum_{i} \sum_{m} \chi_{i, m} v_{i}^{2}} \sqrt{\sum_{m} \sum_{i} \chi_{i, m}\left|g^{\prime}\left(\varphi_{i}\right)\right|^{2} \sum_{j} \chi_{j, m+k+1} v_{j}^{2}} \\
& \quad \leqq \sqrt{K} \sum_{k \geqq 1} \frac{-\varphi^{\prime}(k \delta)}{\sqrt{\varphi((k+2) \delta)}} \sum_{j}\left|v_{j}\right|^{2} .
\end{aligned}
$$

We can suppose $\delta$ is chosen small enough that $\varphi(y+2 \delta)>\frac{1}{2} \varphi(y-\delta)$ for $y>2$, which implies $-\varphi^{\prime}(k \delta) / \sqrt{\varphi((k+2) \delta)}<-4 \varphi^{\prime}(x \delta) / \sqrt{\varphi(x \delta)}$ for $x \in[k-1, k]$, $k>2 / \delta$, and hence that the infinite sum over $k$ above converges. We have the same bound for the contribution of the sum over $j<i$ in (4.5). In view of (2.2) this proves (4.4b). Since $\tilde{U}^{\prime \prime}(x)<C_{\varepsilon}\left|\varphi^{\prime}(x)\right|$, the same proof works for (4.4a). Thus the proof of (4.2) is finished.

From (4.2) it clearly follows that

$$
\lim _{N \rightarrow \infty} \int_{0}^{T} \frac{1}{N} \sum_{i} F_{i}(t) \tilde{F}_{i, \varepsilon}(t) g\left(\varphi_{i}(t) ; K\right) \int_{0}^{1} h_{N}\left(x_{i}(t)-\theta\right) d \theta d t=0,
$$

where $h_{N}(x)=N h(N x)$ as before. The integral under the limit may be written

$$
T \int \sum_{i} \sum_{j \neq i} U^{\prime}\left(q_{i}-q_{j}\right) \sum_{j \neq i} \tilde{U}_{(\varepsilon)}^{\prime}\left(q_{i}-q_{j}\right) g\left(\sum_{j \neq i} \varphi\left(q_{i}-q_{j}\right) ; K\right) h\left(q_{i}\right) \mu^{N}(d \mathbf{q}) .
$$

Hence an application of Corollary 3 shows that for each limit point $\mu$,

$$
\int \sum_{i} \sum_{j \neq i} U^{\prime}\left(q_{i}-q_{j}\right) \sum_{j \neq i} \tilde{U}_{(\varepsilon)}^{\prime}\left(q_{i}-q_{j}\right) g\left(\sum_{j \neq i} \varphi\left(q_{i}-q_{j}\right) ; K\right) h\left(q_{i}\right) \mu(d \mathbf{q})=0 .
$$

Finally, noticing $\int \sum_{i} h\left(q_{i}\right) \mu(d \mathbf{q})<\infty$, we let $\varepsilon \downarrow 0$ to conclude

$$
\int \sum_{i}\left[\sum_{j \neq i} U^{\prime}\left(q_{i}-q_{j}\right)\right]^{2} g\left(\sum_{j \neq i} \varphi\left(q_{i}-q_{j}\right) ; K\right) h\left(q_{i}\right) \mu(d \mathbf{q})=0 .
$$

Since $\sum_{j \neq i} \varphi\left(q_{i}-q_{j}\right)<\infty$ for all $i$ a.s. $(\mu)$ (this simply follows from the fact that $\mu$ is a translation invariant probability on the space of locally finite configurations), letting $K \rightarrow \infty$ in this relation proves $\sum_{j \neq i} U^{\prime}\left(q_{i}-q_{j}\right)=0$ for all $i$ a.s. $(\mu)$. The proof of Lemma 5 is complete.

Theorem 3 immediately follows from Lemma 5 if we prove the next theorem, where neither (1.4) nor (1.5) is needed. 
Theorem 4. Let $\left.\left(\left(q_{i}\right)_{i \in \mathbf{Z}}\right) ; \mu\right)$ be a translation invariant point process on $\mathbf{R}$. If (4.1) holds with $U$ satisfying (1.2) and (1.3), then with $\mu$-probability one either $\left(q_{i}\right)$ has equal successive spacings of a common span less than $R$ or all the spans between nearest-neighbour particles are greater or equal to $R$.

For the proof of Theorem 4 we suppose $\left(q_{i}\right)_{i \in \mathbf{Z}}$ is numbered in an increasing order, i.e., $q_{i}<q_{i+1}$ for all $i \in \mathbf{Z}$, and put $z_{i}=q_{i}-q_{i-1}$. Let $\eta(z), z>0$, be a non-increasing function such that $0<\eta(x)<R$ and

$$
F(\eta(z)) \geqq 3 F(z), \quad \text { where } \quad F(x):=\left|U^{\prime}(x)\right| .
$$

Clearly such a function $\eta$ exists. We prepare a simple lemma.

Lemma 6. Suppose $U$ satisfies (1.2) and (1.3) and let $\eta$ and $z_{i}$ be as above. If $\sum_{j \neq i} U^{\prime}\left(q_{i}-q_{j}\right)=0$ for all $i$, then $\min \left\{z_{i+1}, z_{i-1}\right\} \geqq \eta\left(z_{i}\right)$ for all $i$.

Proof. By applying $\sum_{j \neq i} U^{\prime}\left(q_{i}-q_{j}\right)=0$ first for $i=0$ and then for $i=1$ together with the monotonicity of $F(x)=\left|U^{\prime}(x)\right|, x>0$, we see

$$
\begin{aligned}
& \sum_{j=-2}^{-\infty} F\left(q_{0}-q_{j}\right)+F\left(z_{0}\right)=F\left(z_{1}\right)+\sum_{j=2}^{\infty} F\left(q_{j}-q_{0}\right) \\
& \leqq F\left(z_{1}\right)+\sum_{j=2}^{\infty} F\left(q_{j}-q_{1}\right)=2 F\left(z_{1}\right)+\sum_{j=-1}^{-\infty} F\left(q_{1}-q_{j}\right) \\
& \quad \leqq 3 F\left(z_{1}\right)+\sum_{j=-2}^{-\infty} F\left(q_{0}-q_{j}\right) ;
\end{aligned}
$$

hence $F\left(z_{0}\right) \leqq 3 F\left(z_{1}\right)$, showing $z_{0} \geqq \eta\left(z_{1}\right)$ as desired.

Proof of Theorem 4. Let $\delta$ be an arbitrary positive number. Let $g(x), x \geqq 0$ be a bounded continuous function such that $g(x)=0$ for $x \leqq \delta$ and strictly increasing for $x>\delta$. Then the condition (4.1) implies, in view of Lemma 6, that with $\mu$ probability one

$$
\min \left\{z_{0}, z_{1}, z_{2}\right\} \geqq \eta(\delta) \text { if } g\left(z_{1}\right)>0
$$

and, by symmetry of $F=\left|U^{\prime}\right|$, that with $\mu$-probability one

$$
\begin{aligned}
0= & g\left(z_{1}\right)\left[\sum_{n \neq 1} U^{\prime}\left(q_{1}-q_{n}\right)-\sum_{n \neq 0} U^{\prime}\left(q_{0}-q_{n}\right)\right] \\
= & g\left(z_{1}\right) \sum_{n=2}^{\infty}\left[F\left(q_{n}-q_{1}\right)-F\left(q_{n-1}-q_{0}\right)\right] \\
& -g\left(z_{1}\right) \sum_{n=2}^{\infty}\left[F\left(q_{1}-q_{-n+2}\right)-F\left(q_{0}-q_{-n+1}\right)\right] .
\end{aligned}
$$

From (4.8) it follows that

$$
g\left(z_{1}\right) \sum_{n \neq 1}\left(\left|U^{\prime}\left(q_{1}-q_{n}\right)\right|+\left|U^{\prime}\left(q_{0}-q_{n-1}\right)\right|\right) \leqq \sum_{n \neq 1}\left[\varphi\left(q_{1}-q_{n}\right)+\varphi\left(q_{0}-q_{n-1}\right)\right]
$$


with $\varphi$ a bounded even function having a monotone integrable tail. Since the density $\rho:=\lim _{K+L \rightarrow \infty, K, L \geqq 0}(K+L)^{-1} \#\left\{i \mid-L \leqq q_{i} \leqq K\right\}$ exists a.s. $(\mu)$, we may suppose $\int \rho d \mu<\infty$ (otherwise one has only to restrict $\mu$ on the set $\{\rho<M\}$ and afterwards let $M \rightarrow \infty$ ). This makes the right side of the inequality above (and hence the left side) integrable relative to $\mu$, so that we can change the order of the summation in (4.9) even under the integration by $\mu$, which we now carry out. The translation invariance of $\mu$ then shows that

$$
\begin{aligned}
0= & \sum_{n=2}^{\infty} \int\left[g\left(z_{1}\right)\left(F\left(q_{n}-q_{1}\right)-F\left(q_{n-1}-q_{0}\right)\right)\right. \\
& \left.-g\left(z_{n}\right)\left(F\left(q_{n}-q_{1}\right)-F\left(q_{n-1}-q_{0}\right)\right)\right] \mu(d \mathbf{q}) \\
= & \sum_{n=2}^{\infty} \int\left[g\left(z_{n}\right)-g\left(z_{1}\right)\right]\left[-F\left(q_{n}-q_{1}\right)+F\left(q_{n-1}-q_{0}\right)\right] \mu(d \mathbf{q}) .
\end{aligned}
$$

Since $\left(q_{n}-q_{1}\right)-\left(q_{n-1}-q_{0}\right)=z_{n}-z_{1}$, and since both $g$ and $F$ are monotone, all the integrands have the same sign and hence must vanish a.s. $(\mu)$; in particular $\left[g\left(z_{2}\right)-g\left(z_{1}\right)\right]\left[F\left(z_{2}\right)-F\left(z_{1}\right)\right]=0$ a.s. $(\mu)$. Finally letting the constant $\delta$, on which the support of $g$ depends, tend to zero, we arrive at the conclusion of Theorem 4.

Remark. The same result as Theorem 4 is obtained by Lang [L] under the additional assumption that $\int\left|U^{\prime}\left(z_{0}\right)\right| d \mu<\infty$. The proof given above is essentially the same as his except for the use of Lemma 6 in place of the integrability of $U^{\prime}\left(z_{0}\right)$.

\section{Proof of Theorem 1}

This section is divided into four subsections. In the last one we show that the weak convergence of $\alpha_{t}^{N}$ implies the strong convergence of its mollified local density $\alpha_{t}^{N}\left(h_{N / \ell}(\cdot-\theta)\right)$ as $N \rightarrow \infty$ and $\ell \rightarrow \infty$ in this order. Taking this result for granted we deduce Theorem 1 from Theorems 2 and 3 in the first three subsections.

5.1. First we carry out some calculation to see that our system is essentially of gradient type. This part of the proof is very similar to the corresponding one in [OV]. Let $J$ be a smooth function on the closed interval $[0,1]$. By $(2.1)$ we obtain

$$
\frac{d}{d t} \alpha_{t}^{N}(J)=\sum_{i=1}^{N} J^{\prime}\left(x_{l}(t)\right) v_{i}(t)
$$

and

$$
\begin{aligned}
\frac{d}{d t} \sum J^{\prime}\left(x_{i}\right) v_{i}= & N \sum J^{\prime \prime}\left(x_{i}\right) v_{i}^{2}-N^{2} \sum J^{\prime}\left(x_{i}\right) v_{i}-N \sum J^{\prime}\left(x_{i}\right) B_{N}\left(x_{i}\right) \\
& -N^{2} \sum_{i} J^{\prime}\left(x_{i}\right) \sum_{j \neq i} U^{\prime}\left(N\left(x_{i}-x_{j}\right)\right)
\end{aligned}
$$

as well, where, as before, $B_{N}(x)=N\left[W_{-}^{\prime}(N x)+W_{+}^{\prime}(N(x-1))\right]$ and the time parameter is suppressed. Since $U^{\prime}$ is odd, the last term in (5.2) may be rewritten as

$$
-\frac{1}{2} N^{2} \sum_{i, j(\neq)}\left(J^{\prime}\left(x_{i}\right)-J^{\prime}\left(x_{j}\right)\right) U^{\prime}\left(N\left(x_{i}-x_{j}\right)\right) \text {. }
$$


Notice that $N^{2}$ times the right side of (5.1) appears as the second term on the right side of (5.2), substitute its expression accordingly obtained into (5.1) and then integrate both sides of the resulting equality on a time interval $[0, t]$ and you see

$$
\begin{aligned}
\alpha_{t}^{N}(J)-\alpha_{0}^{N}(J)= & \frac{1}{N} \int_{0}^{t} \sum J^{\prime \prime}\left(x_{i}\right) v_{i}^{2} d s-\left.\frac{1}{N^{2}} \sum J^{\prime}\left(x_{i}\right) v_{i}\right|_{s=0} ^{t} \\
& -\frac{1}{N} \int_{0}^{t} \sum J^{\prime}\left(x_{i}\right) B_{N}\left(x_{i}\right) d s \\
& +\int_{0}^{t} \frac{1}{2 N} \sum_{i, j(\neq)} \frac{J^{\prime}\left(x_{i}\right)-J^{\prime}\left(x_{j}\right)}{x_{i}-x_{j}} \psi\left(N\left(x_{i}-x_{j}\right)\right) d s .
\end{aligned}
$$

By (2.2) and (3.2) the first and the second terms on the right side converge to zero as $N \rightarrow \infty$. Suppose that $J^{\prime}(0)=J^{\prime}(1)=0$. Then the third term also converges to zero due to the assumption (1.6b) (see the estimate (3.3) that is an immediate consequence of (5.3)). If $\psi$ decays for large $x$ sufficiently fast, then the ratio $\left(J^{\prime}\left(x_{i}\right)-J^{\prime}\left(x_{j}\right)\right) /\left(x_{i}-x_{j}\right)$ in the last term might be replaced by $J^{\prime \prime}\left(x_{i}\right)$. Corollary 3 in Sect. 3 ensures such replacement is valid under the assumption (1.4), so that

$$
\alpha_{t}^{N}(J)-\alpha_{0}^{N}(J)=\int_{0}^{t} \frac{1}{2 N} \sum_{i, j(\neq)} \psi\left(N\left(x_{i}(s)-x_{j}(s)\right)\right) J^{\prime \prime}\left(x_{i}(s)\right) d s+o(1),
$$

where $o(1)$ is locally uniform in $t$.

5.2. For each smooth $J$ satisfying $J^{\prime}(0)=J^{\prime}(1)=0$ the family of continuous functions $\left(\alpha_{t}^{N}(J), 0 \leqq t \leqq T\right), N=1,2, \ldots$, is equi-continuous according to Corollary 1 and (5.4). Clearly it is uniformly bounded. Hence it is relatively compact in the space of continuous functions under the topology of uniform convergence. This shows the family of continuous measure-valued functions $\left(\alpha_{t}^{N}, 0 \leqq t \leqq T\right), N=1,2, \ldots$, also is relatively compact; by Corollary 3 any limit point of it is absolutely continuous for each $t$. We can identify the limiting density, $\rho(\theta, t)$ say, and thereby complete the proof of Theorem 1, if we show that the integral on the right side of (5.4) converges to

$$
\int_{0}^{t} \int_{0}^{1} P(\rho(\theta, s)) J^{\prime \prime}(\theta) d \theta d s
$$

since $\rho(\theta, t)$ is then revealed to be a weak solution of the Cauchy problem (1.8) and $(1.10)$ that is unique by virtue of the following theorem. (See also Lemma 8 below.)

Theorem 5. Let $\alpha_{0}$ be a finite Borel measure on [0,1]. Then for every $T>0$ there is at most one non-negative weak solution $\rho=\rho(\theta, t)$ to the problem (1.8) on $(0, T]$ that satisfies the integrability condition $\int_{0}^{T} d t \int_{0}^{1} P(\rho(\theta, t)) d \theta<\infty$ and the initial condition (1.10), as well.

Proof. Theorem 6 in [U] asserts a similar uniqueness for the nonlinear diffusion equation (1.8a) with the periodic boundary condition. Its proof given there is available also for the case of the reflecting boundary condition $(1.8 \mathrm{~b})$ without any alteration except that we here use the fundamental solution of the heat equation (that plays as a test function in the proof) with the reflecting boundary condition instead of the periodic boundary condition. 
5.3. Let $h$ be the same smooth even function as introduced in Sect. 3. Write $h_{K}(\theta):=$ $K h(K \theta)$ for $K>0$ as before. Then for $\ell>0$,

$$
\begin{aligned}
& \int_{0}^{t} \frac{1}{2 N} \sum_{i, j(\neq)} \psi\left(N\left(x_{i}-x_{j}\right)\right) J^{\prime \prime}\left(x_{i}\right) d s \\
& \quad=\int_{0}^{t}\left[\int_{-\ell / N}^{1+\ell / N} \frac{1}{2 N} \sum_{i, j(\neq)} h_{N / \ell}\left(x_{i}-\theta\right) \psi\left(N\left(x_{i}-x_{j}\right)\right) J^{\prime \prime}(\theta) d \theta\right] d s+O\left(\frac{\ell}{N}\right),
\end{aligned}
$$

where $J^{\prime \prime}$ is smoothly extended outside $[0,1]$. Introducing the function

$$
H_{\psi}^{\ell}(\mathbf{q})=\frac{1}{2} \sum_{i, j(\neq)} \psi\left(q_{i}-q_{j}\right) h_{1 / \ell}\left(q_{i}\right), \quad \mathbf{q}=\left(q_{i}\right)_{i=1}^{N},
$$

we can rewrite the inner integral on the right side above in a concise form:

$$
\int_{-\ell / N}^{1+\ell / N} H_{\psi}^{\ell}\left(N \mathbf{x}^{\theta}(s)\right) J^{\prime \prime}(\theta) d \theta
$$

The contribution from the boundary region $[-\ell / N, 0) \cup(1,1+\ell / N]$ to this integral is negligible since $\Psi^{N}(t, i):=\sum_{j \neq i} \psi\left(N\left(x_{i}(t)-x_{j}(t)\right)\right)$ is uniformly integrable as asserted in Corollary 2 of Sect. 3. For the proof of Theorem 1 it therefore suffices to show

$$
\lim _{\ell \rightarrow \infty} \limsup _{m \rightarrow \infty} \int_{0}^{T} d s \int_{0}^{1}\left|H_{\psi}^{\ell}\left(N_{m} \mathbf{x}^{\theta}(s)\right)-P(\rho(\theta, s))\right| d \theta=0,
$$

where $N_{m}$ is an arbitrary subsequence of $\{N\}$ along which $\alpha_{t}^{N}$ converges to $\rho(\theta, t) d \theta$. Again by virtue of the uniform integrability of $\Psi^{N}(t, i)$ we can replace $H_{\psi}^{\ell}(\mathbf{q})$ in (5.6) by

$$
H_{\psi, M}^{\ell}(\mathbf{q})=\frac{1}{2} \sum_{i}\left(\left[\sum_{j \neq i} \psi\left(q_{i}-q_{j}\right)\right] \wedge M\right) h_{1 / \ell}\left(q_{i}\right) .
$$

On the other hand, since $\rho(\theta, s) P(\rho(\theta, s))$ is integrable with respect to $d \theta d s$ on $[0,1] \times[0, T]$ as we will see in Lemma 7 at the end of this section, we can also replace $P(\rho)$ in $(5.6)$ by

$$
P_{M}(\rho):=\left(\left[\sum_{k=1}^{\infty} \psi(k / \rho)\right] \wedge M\right) \rho .
$$

Put

$$
\rho^{\ell}(\mathbf{q}):=\sum h_{1 / \ell}\left(q_{i}\right)=\frac{1}{\ell} \sum h\left(q_{i} / \ell\right) .
$$

Then $P_{M}(\rho(\theta, s))$, in turn, can be replaced by $P_{M}\left(\rho^{N \varepsilon}\left(N \mathbf{x}^{\theta}(s)\right)\right)$ since $\rho^{N \varepsilon}\left(N \mathbf{x}^{\theta}(s)\right)=$ $\alpha_{t}^{N}\left(h_{1 / \varepsilon}(\cdot-\theta)\right) / 2 \varepsilon$ well approximates the limiting density $\rho(\theta, s)$ as $N \rightarrow \infty$ along $N_{m}$ and $\varepsilon \downarrow 0$ in this order. According to Theorem 6 below we can further replace $P_{M}\left(\rho^{N \varepsilon}\left(N \mathbf{x}^{\theta}(s)\right)\right)$ by $P_{M}\left(\rho^{\ell}\left(N \mathbf{x}^{\theta}(s)\right)\right)$. As a consequence of these replacements of functions the problem is reduced to showing that for all $M \geqq 1$,

$$
\lim _{\ell \rightarrow \infty} \limsup _{N \rightarrow \infty} \int_{0}^{T} d s \int_{0}^{1}\left|H_{\psi, M}^{\ell}\left(N \mathbf{x}^{\theta}(s)\right)-P_{M}\left(\rho^{\ell}\left(N \mathbf{x}^{\theta}(s)\right)\right)\right| d \theta=0,
$$


or by rewriting it by means of $\mu^{N}$,

$$
\lim _{\ell \rightarrow \infty} \limsup _{N \rightarrow \infty} \int\left|H_{\psi, M}^{\ell}(\mathbf{q})-P_{M}\left(\rho^{\ell}(\mathbf{q})\right)\right| \mu^{N}(d \mathbf{q})=0
$$

But this follows from the local equilibrium assertion Theorem 3. (Noticing that both $H_{\psi M}^{\ell}(\mathbf{q})$ and $P_{M}\left(\rho^{\ell}(\mathbf{q})\right)$ are the same order as $\rho^{\ell}(\mathbf{q})$ with $M$ fixed, one should accordingly make a truncation argument that may be based on Corollary 3.)

Thus the proof of Theorem 1 is complete if we prove the following

\section{Theorem 6.}

$$
\lim _{\ell \rightarrow \infty} \limsup \limsup _{\varepsilon \downarrow 0} \int_{N \rightarrow \infty}^{T} d s \int_{0}^{1}\left|\rho^{N \varepsilon}\left(N \mathbf{x}^{\theta}(s)\right)-\rho^{\ell}\left(N \mathbf{x}^{\theta}(s)\right)\right| d \theta=0 .
$$

5.4. The proof of Theorem 6 is done by means of the Young measure. Define a finite measure $\pi^{N, \ell}$ on $[0, T] \times[0,1] \times[0, \infty)$ by

$$
\pi^{N, \ell}(d t d \theta d u)=d t d \theta \delta_{\rho^{\ell}\left(N \mathbf{X}^{\theta}(t)\right)}(d u) .
$$

Then the family $\left(\pi^{N, \ell}(d t d \theta d u) ; N=1,2, \ldots, \ell=1,2, \ldots\right)$ constitutes a relatively compact set of measures. Our task was to identify every limit point of $\alpha_{t}^{N}$. For any subsequence of $N$ along which $\alpha_{t}^{N}$ converges, we can choose its subsequence $N^{*}$ such that $\pi^{N, \ell}$ converges along $N^{*}$ for all $\ell$. Hence it would suffice to study limit points of $\pi^{N, \ell}$ along such a subsequence $N^{*}$. Recall that any limit point of $\alpha_{t}^{N}(d \theta)$ possesses a density.

Theorem 7. Let $N^{*}$ be a subsequence of $N$ along which $\pi^{N, \ell}$ converges for all $\ell=$ $1,2, \ldots$ Let $\rho(\theta, t) d \theta$ and $\pi(d t d \theta d u)$ be limit points of $\alpha_{t}^{N}(d \theta)$ and $\pi^{N, \ell}(d t d \theta d u)$, respectively, as first $N \rightarrow \infty$ along $N^{*}$ and then $\ell \rightarrow \infty$. Then the Young measure $\pi(d t d \theta d u)$ is degenerate at $\rho(\theta, t)$, namely $\pi(d t d \theta d u)=d t d \theta \delta_{\rho(\theta, t)}(d u)$.

The deduction of Theorem 6 from Theorem 7 is straightforward, since the second moment $\int u^{2} \pi^{N, \ell}(d t d \theta d u)$ is uniformly bounded according to Corollary 3. (Cf. Eq. (4.5) in [U].) The proof of Theorem 7 is similar to that given in [V] for its Theorem 7.6. The proof given there is modified according to [SU] and [U] so that we can dispense with the entropy technique as applied in [V]. In both [U] and [SU] the fundamental solution on the torus is used in place of a function $g_{N, \varepsilon, \lambda}=h_{N \varepsilon}-h_{\lambda}$ that is used in [V], for simplicity in [U] and since the model is multidimensional in [SU]. This time we use the fundamental solution for the heat equation on the unit interval with reflecting boundary condition not for simplicity but for necessity for dealing with the boundary term.

Proof of Theorem 7. Let $p_{t}(x, y), 0 \leqq x, y \leqq 1, t>0$, be the fundamental solution for the heat equation $\partial_{t} u=\left(\partial^{2} / \partial x^{2}\right) u$ on $[0,1]$ with the reflecting boundary condition. We will apply the following representation of it:

$$
p_{t}(x, y)=\sum_{n=-\infty}^{\infty}\left[g_{t}(-x+y+2 n)+g_{t}(x+y+2 n)\right]
$$


where $g_{t}(x)=(4 \pi t)^{-1 / 2} \exp \left(-x^{2} /(4 t)\right)$. We carry out the same calculation as that leading to (3.1). This time however we compute, instead of $(d / d t) \sum_{i, j(\neq)} G^{\prime}\left(x_{i}-\right.$ $\left.x_{j}\right) v_{i}$,

$$
\frac{d}{d t} \sum_{i, j(\neq)} \partial_{x} p_{\tau}\left(x_{i}, x_{j}\right) v_{i}
$$

with $\tau$ fixed. Here $\partial_{x}$ denotes the partial differentiation with respect to the first argument of $p_{\tau}$. Since $p_{\tau}$ is symmetric (with respect to $x$ and $y$ ),

$$
N \sum_{i, j(\neq)} \partial_{x} p_{\tau}\left(x_{i}, x_{j}\right) v_{i}=\frac{1}{2} \frac{d}{d t} \sum_{i, j(\neq)} p_{\tau}\left(x_{i}, x_{j}\right) .
$$

In place of (3.1) we accordingly obtain

$$
\begin{aligned}
& \frac{1}{2 N^{2}} \int_{0}^{T} \sum_{i, j, k(\neq)}\left[-N U^{\prime}\left(N\left(x_{i}-x_{j}\right)\right)\right]\left[\partial_{x} p_{\tau}\left(x_{i}, x_{k}\right)-\partial_{x} p_{\tau}\left(x_{j}, x_{k}\right)\right] d t \\
& \quad+\frac{1}{N^{2}} \int_{0}^{T} \sum_{i, j(\neq)}\left[-N U^{\prime}\left(N\left(x_{i}-x_{j}\right)\right)\right] \partial_{x} p_{\tau}\left(x_{i}, x_{j}\right) d t \\
& =\left.\frac{1}{N^{3}} \sum_{i, j(\neq)} v_{i} \partial_{x} p_{\tau}\left(x_{i}, x_{j}\right)\right|_{t=0} ^{T}+\left.\frac{1}{2 N^{2}} \sum_{i, j(\neq)} p_{\tau}\left(x_{i}, x_{j}\right)\right|_{t=0} ^{T} \\
& \quad-\frac{1}{2 N^{2}} \int_{0}^{T} \sum_{i, j(\neq)}\left[v_{i}^{2} \partial_{x}^{2} p_{\tau}\left(x_{i}, x_{j}\right)+v_{i} v_{j} \partial_{x} \partial_{y} p_{\tau}\left(x_{i}, x_{j}\right)\right] d t \\
& \quad+\frac{1}{N^{2}} \int_{0}^{T} \sum_{i, j(\neq)} B_{N}\left(x_{i}\right) \partial_{x} p_{\tau}\left(x_{i}, x_{j}\right) d t .
\end{aligned}
$$

Integrate both sides of this identity with respect to $\tau$ on an interval $\left[K^{2} / N^{2}, b\right]$, where $b$ and $K$ are constants. Since

$$
\int_{a}^{b}\left[\partial_{x} p_{\tau}\left(x_{i}, x_{k}\right)-\partial_{x} p_{\tau}\left(x_{j}, x_{k}\right)\right] d \tau=\int_{x_{l}}^{x_{J}}\left[p_{b}\left(u, x_{k}\right)-p_{a}\left(u, x_{k}\right)\right] d u,
$$

two times the first term on the left side then becomes

$$
\frac{1}{N^{2}} \int_{0}^{T} \sum_{i, j, k(\neq)} \psi\left(N\left(x_{i}-x_{j}\right)\right) \frac{1}{x_{i}-x_{j}} \int_{x_{i}}^{x_{j}}\left[p_{b}\left(u, x_{k}\right)-p_{K^{2} / N^{2}}\left(u, x_{k}\right)\right] d u .
$$

We are to see that this is essentially non-negative in the limit as first $N \rightarrow \infty$ and then $b \downarrow 0, K \rightarrow \infty$, by examining the other terms one by one in the following items $0)$ through iv).

$0)$ Since $\partial_{x} p_{\tau}\left(x_{i}, x_{j}\right)$ has the same sign as $U^{\prime}\left(N\left(x_{i},-x_{j}\right)\right)$, the second term on the left side is non-positive.

i) $\left|\int_{a}^{b} \partial_{x} p_{\tau}(x, y) d \tau\right|$ is bounded whenever $0<a<b<1$.

ii) $\sup _{x, y} \int_{0}^{b} p_{\tau}(x, y) d \tau \rightarrow 0$ as $b \downarrow 0$.

iii) Since $\partial_{x} \partial_{y} p_{\tau}(x, y)=(\partial / \partial \tau) p_{\tau}^{o}(x, y)$, where $p_{t}^{o}$ is the fundamental solution of the heat equation with the absorbing boundary condition (as being clear from the Fourier expansion of $\left.p_{\tau}(x, y)\right)$, the contribution of the third term on the right side 
is bounded below by

$$
-\frac{\left\|p_{b}\right\|_{\infty}}{N} \int_{0}^{T} \sum_{i} v_{i}^{2} d t+\frac{1}{N^{2}} \int_{0}^{T} \sum_{i, j(\neq)}\left[v_{i}^{2} p_{K^{2} / N^{2}}\left(x_{i}, x_{j}\right)-v_{i} v_{j} p_{K^{2} / N^{2}}^{o}\left(x_{i}, x_{j}\right)\right] d t .
$$

The first term vanishes as $N \rightarrow \infty$ and the second term is non-negative since $p_{a} \geqq$ $p_{a}^{o}$ and $p_{a}^{o}(x, y)=p_{a}^{o}(y, x)$.

iv) The boundary term vanishes in the limit as $N \rightarrow \infty$ and $b \downarrow 0$ in this order as is verified as follows. By symmetry we have only to look at the contribution of $x_{i} \leqq 1 / 2$. For every $\varepsilon>0$ this part is bounded by

$$
\begin{aligned}
& \frac{1}{N^{2}} \int_{0}^{T} \sum_{i, j(\neq)}\left|B_{N}\left(x_{i}\right)\right| \chi\left(x_{i}<\frac{\varepsilon}{N}\right)\left|\int_{K^{2} / N^{2}}^{b} \partial_{x} p_{\tau}\left(x_{i}, x_{j}\right) d \tau\right| d t \\
& \quad+\left[2 \sup _{x \geqq \varepsilon}\left|W_{-}^{\prime}(x)\right|\right] \frac{1}{N} \int_{0}^{T} \sum_{i, j(\neq)}\left|\int_{K^{2} / N^{2}}^{b} \partial_{x} p_{\tau}\left(x_{i}, x_{j}\right) d \tau\right| d t
\end{aligned}
$$

at least for large $N$. For each $\varepsilon$ the second term is dominated by a constant multiple of

$$
\int_{0}^{T} \int_{0}^{1}\left[\rho^{1}\left(N \mathbf{x}^{\theta}(t)\right)\right]^{2} d \theta d t \times \sup _{x} \sup _{a: 0<a<b} \int_{0}^{1}\left|\int_{a}^{b} \partial_{x} p_{\tau}(x, y) d \tau\right| d y
$$

as is easily shown as in the proof of Lemma 2 in Sect. 3. The first factor is uniformly bounded and the second one converges to zero as $b \downarrow 0$. In view of (3.3) it therefore suffices for our purpose at present to show the following

\section{Lemma 7.}

$$
\lim _{\varepsilon \rightarrow 0} \sup _{0<b<1} \sup _{K \geqq 1} \sup _{N>K / \sqrt{b}} \sup _{0<y<1} \sup _{0<x<\varepsilon / N}\left|\int_{K^{2} / N^{2}}^{b} \partial_{x} p_{\tau}(x, y) d \tau\right|=0 .
$$

Proof. The contribution to $\partial_{x} p_{\tau}$ of the sum over $n \neq 0$ in the representation (5.8) causes no problem so that we have only to compute the one coming from $n=0$, i.e.,

$$
S_{b, K, N}(x, y):=\int_{K^{2} / N^{2}}^{b}\left[g_{\tau}^{\prime}(x-y)+g_{\tau}^{\prime}(x+y)\right] d \tau \text {. }
$$

Observing

$$
\int_{a}^{b} g_{\tau}^{\prime}(z) d \tau=\frac{1}{\sqrt{4 \pi \tau}} \int_{a}^{b} \frac{z}{2 \tau} e^{-z^{2} / 4 \tau} d \tau=\int_{z / \sqrt{b}}^{z / \sqrt{a}} g_{1}(u) d u,
$$

we see that if $y>x$ and $x<\varepsilon / N$, then

$$
S_{b, K, N}(x, y) \leqq 2 \int_{N y / K-\varepsilon / K}^{N y / K+\varepsilon / K} g_{1}(u) d u+\int_{y / \sqrt{b}-\varepsilon / \sqrt{b} N}^{y / \sqrt{b}+\varepsilon / \sqrt{b} N} g_{1}(u) d u,
$$

which with $N>K / \sqrt{b}$ clearly converges to zero as $\varepsilon \rightarrow 0$ uniformly in $y$ as well as in the other variables. The case $y<x$ is easier, since then $|x \pm y| / \sqrt{a} \leqq 2 \varepsilon / K$. 
We have observed that the limit supremum of the formula in (5.9) as $N \rightarrow \infty$ and then $b \downarrow 0, K \rightarrow \infty$ is non-negative, which implies

$$
\begin{aligned}
I_{1} & :=\liminf _{K \rightarrow \infty} \liminf _{N \rightarrow \infty} * \int_{0}^{T} \frac{1}{N^{2}} \sum_{i, j, k(\neq)} \psi\left(N\left(x_{i}-x_{j}\right)\right) \frac{1}{x_{i}-x_{j}} \int_{x_{t}}^{x_{j}} p_{K^{2} / N^{2}}\left(u, x_{k}\right) d u d t \\
& \leqq \limsup _{b \downarrow 0} \limsup _{N \rightarrow \infty} \int_{0}^{T} \frac{1}{N^{2}} \sum_{i, j, k(\neq)} \psi\left(N\left(x_{i}-x_{j}\right)\right) \frac{1}{x_{i}-x_{j}} \int_{x_{i}}^{x_{j}} p_{b}\left(u, x_{k}\right) d u d t=: I_{2},
\end{aligned}
$$

where $*$ indicates that the limit is taken along the subsequence $N^{*}$ specified in Theorem 7. From now on we can proceed as in [U] or [SU]. Here is given an outline. The integrand on the left side is very singular, but we need only a lower bound for it and can apply Fatou's lemma to deduce from Theorem 3,

$$
\int_{0}^{T} d t \int_{0}^{1} d \theta \int_{0}^{\infty} u P(u) \pi_{t, \theta}(d u) \leqq I_{1}<\infty,
$$

where we have applied also the fact that $\pi(d t d \theta d u)$ can be written $d t d \theta \pi_{t, \theta}(d u)$.

As for the right side we first replace the integral under the double limit supremum by $\int d t \int H_{\psi}^{1}\left(N \mathbf{x}^{\theta}(t)\right) N^{-1} \sum p_{b}\left(\theta, x_{k}\right) d \theta$, which readily justified by Corollary 3 and the smoothness of $p_{b}$. Since $p_{b}\left(\theta, \theta^{\prime}\right)$ can be uniformly approximated by a finite sum of products $a_{k}(\theta) b_{k}\left(\theta^{\prime}\right)$ of smooth functions and by Theorem 3 together with the same truncation argument based on Corollary 2 as made just after (5.6) we can further replace the latter by

$$
\int_{0}^{T} d t \int P_{M}\left(\rho^{\ell}\left(N \mathbf{x}^{\theta}(t)\right)\right) \alpha_{t}^{N}\left(p_{b}(\theta, \cdot)\right) d \theta=\int_{0}^{T} \iint P_{M}(u) \alpha_{t}^{N}\left(p_{b}(\theta, \cdot)\right) \pi^{N, \ell}(d t d \theta d u)
$$

if we let $\ell \rightarrow \infty$ and $M \rightarrow \infty$ in this order after letting $N^{*} \rightarrow \infty$. Noticing that $\alpha_{t}^{N}\left(p_{b}(\theta, \cdot)\right)$ approaches $\int p_{b}\left(\theta, \theta^{\prime}\right) \rho\left(\theta^{\prime}, t\right) d \theta^{\prime}$ uniformly in $(\theta, t)$ (with $b$ fixed) and that $\rho(\theta, t)=\int u \pi_{t, \theta}(d u)$ we accordingly conclude

$$
I_{2}=\underset{b \downarrow 0}{\lim \sup _{0}} \int_{0}^{T} \int d t d \theta \int p_{b}\left(\theta, \theta^{\prime}\right) \int u^{\prime} \pi_{t, \theta^{\prime}}\left(d u^{\prime}\right) d \theta^{\prime} \int P(u) \pi_{t, \theta}(d u) .
$$

We can take the limit within the outer double integral sign $\iint d t d \theta$. In fact by applying both the semi-group property and symmetry of $p_{\tau}$ with the help of Fubini's theorem we see that its integrand may be replaced with

$$
F_{b}(t, \theta):=\int u^{\prime} \pi_{t, \theta}^{(b)}\left(d u^{\prime}\right) \int P(u) \pi_{t, \theta}^{(b)}(d u)
$$

where $\pi_{t, \theta}^{(b)}(d u)=\int_{0}^{1} d \eta \pi_{t, \eta}(d u) p_{b / 2}(\theta, \eta)$, and that $F_{b}(t, \theta)$ is uniformly integrable by $d t d \theta$ owing to the integrability result in (5.10) (see [SU]: the argument leading to (7.8) of it). Therefore

$$
I_{2}=\int_{0}^{T} d t \int d \theta \int u^{\prime} \pi_{t, \theta}\left(d u^{\prime}\right) \int P(u) \pi_{t, \theta}(d u) .
$$

It would be standard to deduce $\pi_{t, \theta}(d u)=\delta_{\rho(\theta, t)}(d u)$ from (5.10), (5.11) and the monotonicity of $P$. The proof of Theorem 6 is finished.

By (5.10) and Theorem 6 we incidentally obtain 
Lemma 8. If $\rho(\theta, t) d \theta$ is a limit point of $\alpha_{t}^{N}(d \theta)$, then

$$
\int_{0}^{T} d t \int_{0}^{1} \rho(\theta, t) P(\rho(\theta, t)) d \theta<\infty .
$$

\section{Concluding Remarks}

Remark 1. The function $P(\rho)$ is, at least formally, identified with the pressure in a standard manner based on the virial theorem: indeed $\frac{1}{2} \sum \psi\left(N\left(x_{i}-x_{j}\right)\right)$ is nothing but the interior virial $-\sum q_{i} U^{\prime}\left(q_{i}-q_{j}\right)$ that, since the kinetic energy is negligible, should be balanced with the boundary virial, which in turn must equal the pressure times the volume. This argument however is legitimate only in an equilibrium situation (or in the time average). The following reasoning, though the underlying idea is the same as above, applies to non-equilibrium states. Namely, under the assumption of Theorem 1.1, we deduce from (5.3) that there exist weak limits

$$
p_{-}(t)=\lim _{N \rightarrow \infty} \sum_{i=1}^{N}-W_{-}^{\prime}\left(N x_{i}(t)\right) \quad \text { and } \quad p_{+}(t)=\lim _{N \rightarrow \infty} \sum_{i=1}^{N} W_{+}^{\prime}\left(N x_{i}(t)-N\right)
$$

(the pressure at $\theta=0$ and $\theta=1$, respectively) and that for every smooth function $J$ on $[0,1]$,

$$
\begin{aligned}
\int_{0}^{1} \rho(t, \theta) J(\theta) d \theta-\alpha_{o}(J)= & J^{\prime}(0) \int_{0}^{t} p_{-}(s) d s-J^{\prime}(1) \int_{0}^{t} p_{+}(s) d s \\
& +\int_{0}^{t} \int_{0}^{1} P(\rho(\theta, s)) J^{\prime \prime}(\theta) d \theta d s .
\end{aligned}
$$

This identity with $J$ suitably taken and varied verifies that $p_{-}(t)=P(\rho(0, t))$ and $p_{+}(t)=P(\rho(1, t))$ a.s., where the version of the weak solution $\rho(\theta, t)$ should be properly chosen.

Remark 2. The initial energy bound $(1.9), \mathscr{E}^{N}(0)=o\left(N^{3}\right)$, is natural and indispensable for the conclusion of Theorem 1 to hold. If it is violated, then the socalled initial layer appears in general, i.e., the limiting density of $\alpha_{t}^{N}$, even if it exists and satisfies the non-linear diffusion equation (1.8), can assume an initial trace $\lim _{t \downarrow 0} \rho(\theta, t) d \theta$ different from $\alpha_{0}:=\lim \alpha_{0}^{N}$. For example consider the initial phases $q_{i}(0)=i / 2 ; p_{i}(0)=N / 2, i=1, \ldots, N$. Then within a macroscopically negligible time, e.g., $t=N$, each particle $q_{i}(t)$ travels a distance of about $N / 2$, a macroscopically significant distance of $1 / 2$, which results in the initial discontinuity of the macroscopic mass distribution.

Even if (1.9) does not hold, $\alpha_{t}^{N}$ can still converge uniformly for $t \in[1 / T, T]$ for each $T<\infty$ along suitable subsequences and any limit point may admit a density $\rho(\theta, t)$ that solves (1.8) (though it is then impossible to determine the initial trace from the given datum $\alpha_{0}$ only), which is always the case if

$$
U(x) \leqq c \psi(x) \text { for }|x|<1 \text { and } \mathscr{E}^{N}(0)=O\left(N^{\gamma}\right)
$$

with some constants $c$ and $\gamma(\geqq 3)$. To see this we observe that, in view of (2.2), (3.1), Lemma 3 and Theorem 2, the supplementary assumption on $U$ made above 
implies

$$
\int_{0}^{T} \mathscr{E}^{N}(t) d t \leqq A N+B \frac{\mathscr{E}^{N}(0)}{N^{2}}
$$

for some constants $A$ and $B$. Since $\mathscr{E}^{N}(t)$ is decreasing in $t$, the substitution of $T=N^{-\lambda} \tau$ gives $\tau \mathscr{E}^{N}\left(N^{-\lambda} \tau\right) \leqq A N^{1+\lambda}+B N^{-2+\lambda} \mathscr{E}^{N}(0)$. Applying this inequality, repeatedly according to the magnitude of $\gamma$, we deduce from $\mathscr{E}^{N}(0)=O\left(N^{\gamma}\right)$ that

$$
\mathscr{E}^{N}\left(N^{-\lambda}\right)=O\left(N^{1+\lambda}\right) \text { for } 0<\lambda<2 .
$$

Taking $\lambda=1$, e.g., and applying Theorem 1.1 to the system (2.1) started at $t=$ $N^{-1}$, we obtain the asserted convergence of $\alpha_{t}^{N}$ with a limiting density satisfying (1.8).

Remark 3. If the condition (1.4) fails to hold or equivalently $\int^{\infty} \psi(x) d x=\infty$, then the inner sum on the right side of (1.11) diverges to infinity in the hydrodynamic limit and we need to scale the time by a factor smaller than $N^{2}$ for obtaining a non-trivial limit. A heuristic argument suggests that instead of (1.7) we should define

$$
\begin{array}{ll}
x_{i}(t)=N^{-1} q_{i}\left(\left(N^{-1} \log N\right) t\right) & \text { if } \psi(x) \sim x^{-1} \text { as } x \rightarrow \infty, \\
x_{i}(t)=N^{-1} q_{i}\left(N^{-1+\gamma} t\right) & \text { if } \psi(x) \sim x^{-1+\gamma} \text { as } x \rightarrow \infty,
\end{array}
$$

where $0<\gamma<1$ : in the former case we would have Eq. (1.8) with $P(\rho)=\rho^{2}$ for the limiting density field, while in the latter case (1.8) must be replaced by

$$
\frac{\partial}{\partial t} \rho(\theta, t)=\frac{\partial}{\partial \theta}\left[\rho(\theta, t) \int \frac{\rho(\theta+u, t)-\rho(\theta-u, t)}{u}|u|^{-\gamma} d u\right] \text {. }
$$

\section{References}

[A] Aronson, D.G.: The porous medium equation. In: Nonlinear Diffusion Problems. A. Fasano, M. Primicerio (eds), Vol. 1224, Springer Lecture Notes in Math., Berlin, Heidelberg, New York: Springer, 1986

[BDS] Boldrighini, C., Dobrushin, R.N., Suhov, Yu.M.: One-dimensional hard rod caricature of hydrodynamics. J. Stat. Phys. 31, 577-615 (1983)

[F] Fritz, J.: On the asymptotic behaviour of Spitzer's model for evolution of one-dimensional point systems. J. Stat. Phys. 38, 615-645 (1985)

[GPV] Guo, M.Z., Papanicolaou, G.C., Varadhan, S.R.S.: Non-linear diffusion limit for a system of nearest neighbor interactions. Commun. Math. Phys. 118, 31-59 (1988)

[L] Lang, R.: On the asymptotic behaviour of infinite gradient systems. Commun. Math. Phys. 65, 129-149 (1979)

[M] Mürmann, M.G.: The hydrodynamic limit of a one-dimensional nearest neighbor gradient system. J. Stat. Phys. 48, 769-788 (1987)

[OV] Olla, S., Varadhan, S.R.S.: Scaling limit for interacting Ornstein-Uhlenbeck processes. Commun. Math. Phys. 135, 355-378 (1991)

[PS] Presutti, E., Scacciatelli, E.: The evolution of a one-dimensional point system: A note on Fritz's paper. J. Stat. Phys. 38, 647-653 (1985)

[S] Sinai, Ya.G.: Dynamics of local equilibrium Gibbs distributions and Euler equations. The one-dimensional case. Selecta Math. Sov. 7, 279-289 (1988)

[SU] Suzuki, Y., Uchiyama, K.: Hydrodynamic limit for a spin system on a multidimensional lattice. Probab. Theory Relat. Fields 95, 47-74 (1993) 
[U] Uchiyama, K.: Scaling limits of interacting diffusions with arbitrary initial distributions. Probab. Theory Relat. Fields 99, 97-110 (1994)

[V] Varadhan, S.R.S.: Scaling limit for interacting diffusions. Commun. Math. Phys. 135, 313 $353(1991)$

Communicated by J.L. Lebowitz 